\title{
Regional Continuous Quality Improvement in Cardiopulomary Resuscitation optimizing the local "Chain of Survival"
}

KJETIL SUNDE ( $\square)$ Surgical Intensive Care Unit Oslo University Hospital Ulleval $\mathrm{N}-0407$ Oslo,Norway Phone: +4723016787 Fax: +4723016799 E-mail: kjetil.sunde@medisin.uio.no

\author{
KJETIL SUNDE
}

This review article is based on a recent published paper from our group: Lund-Kordahl I, Olasveengen TM, Lorem T, Samdal M, Wik L, Sunde K. Improving outcome after out-of-hospital cardiac arrest by strengthening weak links of the local Chain of Survival; quality of advanced life support and post-resuscitation care.

Resuscitation 2010;81:422-6(1).

\begin{abstract}
Survival after out-of-hospital cardiac arrest (OHCA) depends on a well functioning Chain of Survival. In tihis article we analysed different phases of process of improving outcome after OHCA: recognition of potential for improved survival within the local system and strictly implementation guidelines for elimination weak links. Continuous focus on the importance of a well functioning Chain of Survival with specific strategies to improve quality of care during advanced life support (ALS) and post resuscitation has lead to significantly improved outcome of patients with OHCA in Oslo.
\end{abstract}

Keywords: cardiac arrest, local Chain of Survival, improving outcome, weak links, Oslo, three different time periods

\section{Introduction}

Approximately 275000 Europeans suffer from out-of- hospital cardiac arrest (OHCA) and are treated by the local Emergency Medical Systems (EMS). (2) Despite evolving evidence based guidelines for cardiopulmonary resuscitation (CPR), $(3,4)$ survival rates after OHCA has not improved much in the majority of places around the world. In Europe, average survival rate was recently reported to be around $10 \%$, but with a huge spread between 5 and $20 \%$. (2) This difference in successful outcome is mainly due to the overall quality of the local Chain of Survival; early arrest recognition and call for help early CPR, early defibrillation and early post resuscitation care. (5)

Whereas CPR and defibrillation have received the most attention and been the major research areas the present three decades, post resuscitation care has the last 5-10 years received much more focus. And, a focused, goaldirected treatment strategy throughout the local Chain of Survival is of utmost importance. Successful survival (defined as a survived patient with a normal to just a slighly reduced neurological function, independent, back to normal life activites and work) is totally dependent on a well functioning preand inhospital system of care. This is very similar and highly comparable to success in sports; you will only win the gold medal in a $4 \times 100$ relay in running if all the four athlets are in excellent condition, good prepared and with an excellent collaboration and switching in between the different stages. In other words, all stages, such as early recognication, initiation of bystander CPR, activation of the EMS system, good quality Basic Life Support (BLS), early defibrillation, good quality Advanced Life Support (ALS), and finally, goaldirected, standardised good quality post resuscitation care including percutaneous coronary intervention $(\mathrm{PCl})$ and therapeutic hypothermia $(\mathrm{TH})$ are all important steps for a successful outcome. And, the switch between the different treatment steps must be smooth and well organised and prepared. 


\section{Optimizing the local "Chain of Survival"}

How to locally improve survival after OHCA? First of all, documenting Utstein data for OHCA is a very useful tool for evaluating and critically assessing the local Chain of Survival, and for identification of areas in need of improvements. (6) Then, after critical evaluation of the local Utstein data, two important questions have to be asked:

Are we happy with the survival rate within our institution?

If not, where are the weak links?

So, to emphasize, the most important step is to document and understand that there is a potential for improved survival within the local system. Further, the weak links, partly responsible for this bad outcome, have to be identified and challenged. Efforts to improve the weakest links have to be initiated and strict implemented. The system will never get real good if the weak links stay weak!

\section{Regional Continuous Quality Improvement in Cardiopulomary Resuscitation in Oslo}

In Oslo, Norway, survival to hospital discharge for OHCA of cardiac origin has been below $10 \%$ for the last 30 years. (7-9) The city of Oslo covers $454 \mathrm{~km}^{2}$ and has approximately 550 000 inhabitants. Oslo has a one-tiered community run EMS system. All acute care ambulances are manned with paramedics. On weekdays between 7:30 and 22:00, a physician-manned ambulance staffed by two paramedics and an anesthesiologist functions on the same level as the regular paramedic staffed ambulances. All paramedics are trained to use defibrillators in manual mode. Endotracheal intubation is the standard method for securing the airways, followed by uninterrupted chest compressions with 10-12 interposed ventilations per minute. There are several hospitals taking care of OHCA patients, but the majority of the patients $(60-70 \%)$ are admitted to a 24 hours $\mathrm{PCl}$ center; Oslo University Hospital Ulleval.
We recently published a paper based on a study evaluating survival after OHCA from Oslo over a ten year period, with some important changes in the local Chain of Survival implemented at different time periods. (1) During the first period (1996-98), ambulance personnel provided ALS according to the 1992 Guidelines, (10) resulting in poor ALS quality with long periods without vital organ perfusion and few shocks resulting in return of spontaneous circulation (ROSC). (8) There was no standardised post resuscitation care/treatment plan at any of the five receiving hospitals at that time. Modified 2000 Guidelines, (11) with more focus on chest compression quality and three minutes of CPR prior to and between defibrillation were implemented prior to the second period (2001-2003), based on findings from some local ALS studies. $(8,12)$ The main aim of these changes was to optimise vital organ perfusion by minimizing interruptions in chest compression and a more reasonable timing of defibrillations, defined as improving the third link in our local Chain of Survival. However, there was still no standardised post resuscitation care in the inhospital part of our local chain. In the third period (2004-2005), there were no additional changes in the prehospital ALS protocol, but standardised post resuscitation care protocols had finally been implemented in the Oslo hospitals. (13) At this time, we had realised that quality of hospital care after cardiac arrest matters, (14-16) and the landmark studies on therapeutic hypothermia had just been published. $(14,15)$ In a study from Norway, we documented that survival to discharge varied between $34 \%$ and $56 \%$ in four different hospital regions. (16) In similar, a recent US report showed a difference in mortality from $41-81 \%$ among 39 institutions. (17) The post resuscitation protocols were applied to all patients regardless of initial rhythm or aetiology if active treatment was desired. (13) If coronary angiography and/or $\mathrm{PCl}$ was indicated, patients were directly transported from the scene to one of the two hospitals with this capacity (24 hours/day).
Our local Chain of Survival was thereby changed throughout this ten year period, with special focus on the last two weakest links. The first period (199698) had poor ALS quality and post resuscitation care, the second period (2001-2003) had good ALS quality but poor post resuscitation care, and the third period (2004-2005) had both good ALS quality and post resuscitation care. During these three different time periods, altogether 1320 patients received ALS for OHCA. (1)

The results showed that there was a significantly improved overall survival to hospital discharge across the three time periods, from $7 \%$ to $10 \%$ and $13 \%$ $(p=0.001)$. (1) Survival with favorable neurological outcome (cerebral performance cathegory CPC 1-2) was also significantly improved $(6 \%, 9 \%$ and $12 \%, p=0.001)$. OHCA patients with cardiac origin had also significantly improvement in survival, from $9 \%$ to $12 \%$ and $17 \%$, respectively $(p=0.003)$. And, finally, among those patients with the best survival potential, bystander witnessed cardiac arrests with cardiac aetiology and initial ventricular fibrillation (VF), survival with favorable neurological outcome was 14\%, 22\% and 33\% $(p=0.001)$, respectively. Interestingly, we documented that despite an increase in negative prognostic factors such as more unwitnessed non-VF arrests and increased response intervals, outcome was doubled during these 10 years. (1)

This has been achieved trough improvents in quality of ALS and post-resuscitation care. With high rates of bystander CPR in our EMS (above 50\%), (7-9) the last two links were considered to be our weakest. Although none of the ALS components such as drug administration, intubation or advanced airway management have been documented to improve survival, $(18,19)$ quality of ALS is still of course important for outcome after cardiac arrest. However, besides early defibrillation, quality of good CPR/chest compressions is probably more important than drugs (18) or other interventions/devices. During implementation of the modified 2000 
guidelines, our ALS training thereby focused on optimising chest compressions by stressing the importance of adequate force and rate as well as limiting hands-off intervals. Recent studies documenting the quality of chest compressions in our EMS $(18,20,21)$ have demonstrated huge improvements compared to chest compression quality prior to implementation of the 2000 guidelines. (8)

The increase in survival, however, was most prominent for the third time period, demonstrating the importance of improved post resuscitation care. After implementation of our standardised post-resuscitation protocol including $\mathrm{TH}$, frequent use of coronary angiography/PCl, early optimisation of haemodynamics, metabolism and seizure control, favourable survival rates among patients with witnessed VF arrests admitted to ICU increased from $34 \%$ in the first period to $56 \%$ in the third period. (13) Similar good results have also been documented by others, $(22,23)$ and the implementation part of new treatment strategies like fro example $\mathrm{TH}$ is challenging and of utmost importance. (24)

Our findings are in accordance with recent studies reporting temporal improvements in survival within two different EMS systems. $(25,26)$ A study from Seattle reported cumulative effect of four EMS program changes with overall survival to hospital discharge improving from $31 \%$ to $45 \%$ during a 30 year period in patients with witnessed VF arrest. (25) Their local Chain of Survival was strengthened by focusing on the second and third links with strategies to provide early BLS through telephone assisted CPR and early defibrillation through public access defibrillators and equipping first responders with defibrillators. (25) Similarly, a large-scale population-based study from Japan reported improved survival during an eight year period after implementing changes to the first three links in their local chain of survival. (26) By training lay persons in BLS, introducing telephone assisted CPR, placing public access defibrillators and training emergency medical technicians in defibrillation, tracheal intubation and administration of epinephrine, they saw increased neurological intact survival (from 6\% to $16 \%$ ) for patients with witnessed VF arrest. (26)

Continuous focus on the importance of a well functioning Chain of Survival with specific strategies to improve quality of care during ALS and post resuscitation has lead to significantly improved out- come of patients with OHCA in Oslo. In order to continuously improve outcome from cardiac arrest in any EMS system, attention should be paid to all aspects of the Chain of Survival. Resuscitation Outcomes Consortium Investigators have recently published that there are large regional variation in OHCA incidence and outcome, (27) and in a previous review overall outcome for OHCA was reported to vary between $2-11 \%$ for EMS systems with median response intervals of 8-9 minutes. (28) With a response interval of 9 minutes, outcome for our EMS has moved from mid-range to top-end of this scale. Such system variations may make a huge impact on results from multicenter intervention clinical trials, and institutions should be encouraged to identify and improve weak links in their Chain of Survival to optimize quality of care before new interventions are introduced and investigated. In Maribor, the second largest city of Slovenia, they have shown the survival potential after OHCA; in their effective and innovative resuscitation system, $(29,30)$ they achieve survival rates for all rhythms from $21-26 \%$ the past 5 years (dr. Stefek Grmec). These excellent results should be targeted goals for everyone interested in improving outcome after OHCA! It is possible!

\section{REFERENCES}

1.Lund-Kordahl I, Olasveengen TM, Lorem T, Samdal M, Wik L, Sunde K. Improving outcome after out-of-hospital cardiac arrest by strengthening weak links of the local Chain of Survival; quality of advanced life support and post-resuscitation care. Resuscitation 2010;81:422-6. 2.Atwood C, Eisenberg MS, Herlitz J, Rea TD. Incidence of EMS-treated out-of-hospital cardiac arrest in Europe. Resuscitation 2005;67:75-80. 3.2005 American Heart Association Guidelines for Cardiopulmonary Resuscitation and Emergency Cardiovascular Care. Circulation 2005;112:IV-1-IV-203

4.European Resuscitation Council Guidelines for Resuscitation 2005. Resuscitation 2005;67:S3-S180.

5.Cummins RO, Ornato JP, Thies WH, Pepe PE. Improving survival from sudden cardiac arrest: the "chain of survival" concept. A statement for health professionals from the Advanced Cardiac Life Support Subcommittee and the Emergency Cardiac Care Committee, American Heart Association. Circulation 1991;83:1832-47.

6. Jacobs I, Nadkarni V, Bahr J,Berg RA, Billi JE, Bossaert L, et al. Cardiac arrest and cardiopulmonary resuscitation outcome reports: update and simplification of the Utstein templates for resuscitation registries: a statement for healthcare professionals from a task force of the International Liaison Committee on Resuscitation (American Heart Association, European Resuscitation Council, Australian Resuscitation Council, New Zealand Resuscitation Council, Heart and Stroke Foundation of Canada, InterAmerican Heart Foundation, Resuscitation Councils of Southern Africa). Circulation 2004;110:3385-97. 
7.Lund I, Skulberg A. Cardiopulmonary resuscitation by lay people. Lancet 1976;2:702-4.

8.Sunde K, Eftestol T, Askenberg C, Steen PA. Quality assessment of defribrillation and advanced life support using data from the medical control module of the defibrillator. Resuscitation 1999;41:237-47.

9.Naess AC,.Steen PA. Long term survival and costs per life year gained after out-of-hospital cardiac arrest. Resuscitation 2004;60:57-64.

10.Guidelines for advanced life support. A statement by the Advanced Life Support Working Party of the European Resuscitation Council, 1992. Resuscitation 1992;24:111-21.

11.Guidelines 2000 for cardiopulmonary resuscitation and emergency cardiovascular care - An international consensus on science. Resuscitation 2000;46:3-437.

12.Wik L, Hansen TB, Fylling F, Steen T,Vaagenes P, Auestad BH, et al. Delaying defibrillation to give basic cardiopulmonary resuscitation to patients with out-of-hospital ventricular fibrillation: a randomized trial. JAMA 2003;289:1389-95.

13.Sunde K, Pytte M, Jacobsen D, Mangschau A, Jensen LP, Smersrud C, et al. Implementation of a standardised treatment protocol for post resuscitation care after out-of-hospital cardiac arrest. Resuscitation 2007;73:29-39.

14.The Hypothermia After Cardiac Arrest (HACA) study group: Mild TH to improve the neurologic outcome after cardiac arrest. New Engl J Med 2002;346:549-56.

15.Bernard SA, Gray TW, Buist MD, Jones BM, Silvester W, Gutteridge G,et al. Treatment of comatose survivors of out-of-hospital cardiac arrest with induced hypothermia. New Engl J Med 2002;346:557-63.

16.Langhelle A, Tyvold SS, Lexow K, Hapnes S, Sunde K, Steen PA. In-hospital factors associated with improved outcome after out-of-hospital cardiac arrest. A comparison between four regions in Norway. Resuscitation 2003;56:247-63.

17.Carr BG, Kahn JM, Merchant RM, Kramer AA, Neumar RW. Inter-hospital variability in post-cardiac arrest mortality. Resuscitation 2009;80:30-4.

18. Olasveengen TM, Sunde K, Brunborg C, Thowsen J, Steen PA, Wik L. Intravenous drug administration during out-of-hospital cardiac arrest: a randomized trial. JAMA 2009 Nov 25;302:2222-9.

19.Stiell IG, Wells GA, Field B, Spaite DW, Nesbitt LP, De Maio VJ, et al. Advanced Cardiac Life Support in Out-of-Hospital Cardiac Arrest. N Engl J Med 2004;351:647-56.

20. Olasveengen TM, Wik L, Kramer-Johansen J, Sunde K, Pytte M, Steen PA. Is CPR quality improving? A retrospective study of out-of-hospital cardiac arrest. Resuscitation 2007;75:260-6.

21. Olasveengen TM, Vik E, Kuzovlev A, Sunde K. Effect of implementation of new resuscitation guidelines on quality of cardiopulmonary resuscitation and survival. Resuscitation 2009;80:407-11.

22. Oddo M, Schaller MD, Feihl F, Ribordy V, Liaudet L. From evidence to clinical practice: effective implementation of therapeutic hypothermia to improve patient outcome after cardiac arrest. Crit Care Med 2006;34:1865-73.

23. Knafelj R, Radsel P, Ploj T, Noc M. Primary percutaneous coronary intervention and mild induced hypothermia in comatose survivors of ventricular fibrillation with ST-elevation acute myocardial infarction. Resuscitation 2007;74: 227-34.

24.Soreide E, Sunde K. TH after out-of hospital cardiac arrest: how to secure world-wide implementation? Curr Opin Anesth 2008;21:209-15.

25.Becker L, Gold LS, Eisenberg M, White L, Hearne T, Rea T. Ventricular fibrillation in King County, Washington: A 30-year perspective. Resuscitation 2008;79:22-7.

26.Iwami T, Nichol G, Hiraide A, Hayashi Y, Nishiuchi T, Kajino K, et al. Continuous improvements in "chain of survival" increased survival after out-of-hospital cardiac arrests: a large-scale population-based study. Circulation 2009;119:728-34.

27. Nichol G, Thomas E, Callaway CW,Hedges J, Powell JL, Aufderheide TP, et al. Regional variation in out-of-hospital cardiac arrest incidence and outcome. JAMA 2008;300:1423-31.

28.Fredriksson M, Herlitz J, Nichol G. Variation in outcome in studies of out-of-hospital cardiac arrest: a review of studies conforming to the Utstein guidelines. Am J Emerg Med 2003;21:276-81.

29.Grmec S, Krizmaric M, Mally S, Kozelj A, Spindler M, Lesnik B. Utstein style analysis of out-of-hospital cardiac arrest--bystander CPR and end expired carbon dioxide. Resuscitation 2007;72:404-14.

30.Grmec S, Strnad M, Kupnik D, Sinkovic A, Gazmuri RJ. Erythropoietin facilitates the return of spontaneous circulation and survival in victims of out-of-hospital cardiac arrest. Resuscitation 2009;80:631-7. 